\title{
ERRATUM
}

\section{A cohesive finite element for quasi-continua}

\author{
Xiaohu Liu - Shaofan Li • Ni Sheng
}

Published online: 2 July 2008

(c) Springer-Verlag 2008

\section{Erratum to: Comput Mech}

\section{DOI 10.1007/s00466-007-0222-6}

We have found the following typos and errors in the article, A cohesive finite element for quasi-continua:

1. Page 2, Eq. (5),

$\mathbf{P}^{+} \cdot \mathcal{N}^{+}=\mathbf{P}^{-} \cdot \mathcal{N}^{-}$on $S_{0}^{ \pm}$,

should change to

$\mathbf{P}^{+} \cdot \mathcal{N}^{+}+\mathbf{P}^{-} \cdot \mathcal{N}^{-}=0 \quad$ on $S_{0}^{ \pm}$.

2. Page 2, Eq. (7),

$$
\begin{aligned}
\int_{B_{0}^{ \pm}} & \rho_{0} \ddot{\boldsymbol{\varphi}} \cdot \delta \boldsymbol{\varphi} d V+\int_{B_{0}^{ \pm}} \mathbf{P}(\boldsymbol{\varphi}) \cdot \delta \mathbf{F} d V \\
= & \int_{\partial_{t} B_{0}^{ \pm}} \overline{\mathbf{t}} \cdot \delta \boldsymbol{\varphi} d S+\int_{S_{0}}(\mathbf{P}(\boldsymbol{\varphi}) \cdot \mathbf{N}) \\
& \cdot \delta\left(\boldsymbol{\varphi}^{+}-\boldsymbol{\varphi}^{-}\right) d S,
\end{aligned}
$$

should change to

$$
\begin{aligned}
& \int_{B_{0}^{ \pm}} \rho_{0} \ddot{\boldsymbol{\varphi}} \cdot \delta \boldsymbol{\varphi} d V+\int_{B_{0}^{ \pm}} \mathbf{P}(\boldsymbol{\varphi}): \delta \mathbf{F} d V \\
& \quad=\int_{\partial_{t} B_{0}^{ \pm}} \overline{\mathbf{t}} \cdot \delta \boldsymbol{\varphi} d S+\int_{S_{0}}(\mathbf{P}(\boldsymbol{\varphi}) \cdot \mathcal{N}) \cdot \delta\left(\boldsymbol{\varphi}^{+}-\boldsymbol{\varphi}^{-}\right) d S .
\end{aligned}
$$

The online version of the original article can be found under doi:10.1007/s00466-007-0222-6.

X. Liu $\cdot$ S. Li $(\varangle) \cdot$ N. Sheng

Department of Civil and Environmental Engineering, University of California, Berkeley, CA 94720, USA

e-mail: shaofan@berkeley.edu; li@ newton.berkeley.edu
3. Page 5, Eq. (32),

$\mathbf{f}^{\text {cohe }}=\int_{S_{0}} \frac{\partial W^{S}}{\partial \boldsymbol{\Delta}^{d}}\left[\mathbf{N}^{S}\right]^{T} d S$,

should change to

$\mathbf{f}^{\text {cohe }}=\int_{S_{0}} \frac{\partial W^{s}}{\partial \boldsymbol{\Delta}}\left[\mathbf{N}^{S}\right]^{T} d S$.

4. Page 6, Eq. (42),

$$
\begin{aligned}
\frac{\partial \mathbf{r}^{i j}}{\partial \boldsymbol{\Delta}^{d}} & =\frac{\partial \boldsymbol{\Delta}}{\partial \boldsymbol{\Delta}^{d}}+\frac{\partial \mathbf{h}^{+}}{\partial \boldsymbol{\Delta}^{d}}-\frac{\partial \mathbf{h}^{-}}{\partial \boldsymbol{\Delta}^{d}} \\
& =\mathbf{N}^{s}+\frac{\partial \mathbf{h}^{+}}{\partial \mathbf{d}^{+}} \frac{\partial \mathbf{d}^{+}}{\partial \boldsymbol{\Delta}^{d}}-\frac{\partial \mathbf{h}^{-}}{\partial \mathbf{d}^{-}} \frac{\partial \mathbf{d}^{-}}{\partial \boldsymbol{\Delta}^{d}} \\
& =\mathbf{N}^{s}+\frac{1}{2} \mathbf{B}^{+} \mathbf{H}^{+}-\frac{1}{2} \mathbf{B}^{-} \mathbf{H}^{-}
\end{aligned}
$$

should change to

$$
\begin{aligned}
\frac{\partial \mathbf{r}^{i j}}{\partial \boldsymbol{\Delta}^{d}} & =\frac{\partial \boldsymbol{\Delta}}{\partial \boldsymbol{\Delta}^{d}}+\frac{\partial \mathbf{h}^{+}}{\partial \boldsymbol{\Delta}^{d}}-\frac{\partial \mathbf{h}^{-}}{\partial \boldsymbol{\Delta}^{d}} \\
& =\mathbf{N}^{s}+\frac{\partial \mathbf{h}^{+}}{\partial \mathbf{d}^{+}} \frac{\partial \mathbf{d}^{+}}{\partial \boldsymbol{\Delta}^{d}}-\frac{\partial \mathbf{h}^{-}}{\partial \mathbf{d}^{-}} \frac{\partial \mathbf{d}^{-}}{\partial \boldsymbol{\Delta}^{d}} \\
& =\mathbf{N}^{s}+\frac{1}{2} \mathbf{B}^{+} \mathbf{H}^{+}+\frac{1}{2} \mathbf{B}^{-} \mathbf{H}^{-}
\end{aligned}
$$

5. Page 6, Eq. (43),

$$
\begin{aligned}
\frac{\partial W^{e s}}{\partial \boldsymbol{\Delta}^{d}}= & \frac{N_{e s}}{2 A_{e s}} \sum_{j=1}^{M_{s}} E_{i j}^{\prime}\left(r^{i j}\right) \frac{\partial r^{i j}}{\partial \mathbf{\Delta}^{d}}=\frac{N_{e s}}{2 A_{e s}} \sum_{j=1}^{M_{s}} E_{i j}^{\prime}\left(r^{i j}\right) \frac{1}{r^{i j}} \\
& \times\left(\mathbf{r}^{i j} \cdot \mathbf{N}^{s}+\frac{1}{2} \mathbf{r}^{i j} \cdot \mathbf{B}^{+} \mathbf{H}^{+}-\frac{1}{2} \mathbf{r}^{i j} \cdot \mathbf{B}^{-} \mathbf{H}^{-}\right)
\end{aligned}
$$


should change to

$$
\begin{aligned}
\frac{\partial W^{e s}}{\partial \boldsymbol{\Delta}^{d}}= & \frac{N_{e s}}{2 A_{e s}} \sum_{j=1}^{M_{s}} E_{i j}^{\prime}\left(r^{i j}\right) \frac{\partial r^{i j}}{\partial \boldsymbol{\Delta}^{d}}=\frac{N_{e s}}{2 A_{e s}} \sum_{j=1}^{M_{s}} E_{i j}^{\prime}\left(r^{i j}\right) \frac{1}{r^{i j}} \\
& \times\left(\mathbf{r}^{i j} \cdot \mathbf{N}^{s}+\frac{1}{2} \mathbf{r}^{i j} \cdot \mathbf{B}^{+} \mathbf{H}^{+}+\frac{1}{2} \mathbf{r}^{i j} \cdot \mathbf{B}^{-} \mathbf{H}^{-}\right) .
\end{aligned}
$$

6. Page 6, Eq. (44),

$$
\begin{aligned}
\frac{\partial W^{e s}}{\partial \boldsymbol{\Delta}^{d}}= & \frac{N_{e s}}{2 A_{e s}} \sum_{j=1}^{M_{s}} \frac{E_{i j}^{\prime}\left(r^{i j}\right)}{r^{i j}} \\
& \times\left(\left[\mathbf{N}^{s T}\right] \mathbf{r}^{i j}+\frac{1}{2}\left[\mathbf{B}^{+} \mathbf{H}^{+}\right]^{T} \mathbf{r}^{i j}-\frac{1}{2}\left[\mathbf{B}^{-} \mathbf{H}^{-}\right]^{T} \mathbf{r}^{i j}\right),
\end{aligned}
$$

should change to

$$
\begin{aligned}
\frac{\partial W^{e s}}{\partial \boldsymbol{\Delta}^{d}}= & \frac{N_{e s}}{2 A_{e s}} \sum_{j=1}^{M_{s}} \frac{E_{i j}^{\prime}\left(r^{i j}\right)}{r^{i j}} \\
& \times\left(\left[\mathbf{N}^{s T}\right] \mathbf{r}^{i j}+\frac{1}{2}\left[\mathbf{B}^{+} \mathbf{H}^{+}\right]^{T} \mathbf{r}^{i j}+\frac{1}{2}\left[\mathbf{B}^{-} \mathbf{H}^{-}\right]^{T} \mathbf{r}^{i j}\right) .
\end{aligned}
$$

7. Page 6, Remark 2,

"From (43), we observe that when $\mathbf{B}^{+} \mathbf{H}^{+}=\mathbf{B}^{-} \mathbf{H}^{-}$, the expression of nodal traction will reduce to:" should change to

"If $\mathbf{F}^{+} \approx \mathbf{F}^{-}, \mathbf{F}^{+} \mathbf{H}^{+}-\mathbf{F}^{-} \mathbf{H}^{-} \approx 0$, because $\mathbf{H}^{+}=\mathbf{H}^{-}$. Then the expression of nodal traction vector will reduce to:". 